\title{
Caracterización de las Distribuciones de Energía Cinética en los lones Producidos por Ablación Láser de Metales
}

\section{Jon I. Apiñániz ${ }^{1}$, Roberto Martínez ${ }^{2}$, Asier Longarte ${ }^{1}$ y Fernando Castaño ${ }^{1^{*}}$}

(1) Universidad del País Vasco, Facultad de Ciencia y Tecnología, Departamento de Química Física, Campus de Leioa, Barrio Sarriena s/n, 48940 Leioa (Vizcaya)-España (e-mail: f.castano@ehu.es)

(2) Universidad del País Vasco, Facultad de Farmacia, Departamento de Química Física, Paseo de la Universidad 7, 01006 Vitoria-Gasteiz (Alava)-España.

*autor a quien debe ser dirigida la correspondencia

\section{Resumen}

Se han determinado las distribuciones de energía cinética de los iones producidos por ablación láser pulsada de nanosegundos, sobre Al, $\mathrm{Cu}$ y $\mathrm{Fe}$ a $532 \mathrm{~nm}$ en el vacío. El sistema experimental utilizado es un espectrómetro de masas dotado de un analizador electrostático de energías y un tubo de vuelo. Los perfiles de energía obtenidos se ajustan a distribuciones de Maxwell-Boltzmann con una componente de Coulomb debida a la aceleración de los iones por el intenso campo eléctrico producido tras la emisión de electrones. A fluencias bajas de láser las distribuciones son sencillas, pero a fluencias elevadas el comportamiento es complejo, con recombinaciones e ionizaciones. El estudio de estas distribuciones permite obtener una valiosa información acerca de los procesos que dan lugar a la formación de los iones y de las mejores condiciones para obtener haces de iones de energía cinética predeterminada con fines tecnológicos.

Palabras clave: ablación láser, plasmas, energías cinéticas, ionización de metales, iones

\section{Characterization of Kinetic Energy Distributions of Ions Produced by Laser Ablation Of Metals}

\begin{abstract}
Kinetic energy distributions have been determined for ions produced by laser ablation with $532 \mathrm{~nm}$ nanosecond pulses on $\mathrm{Al}, \mathrm{Cu}$ and $\mathrm{Fe}$ in vacuum. The experiments were carried out in a mass spectrometer that includes an electrostatic energy analyzer and a flight tube. The energy profiles obtained fit Maxwell-Boltzmann distributions with a Coulomb component, which reveals that ions are accelerated by the intense electric field produced after electrons are emitted. While at low laser fluences simple distributions are observed, the behaviour is more complex at higher fluences, due to the presence of recombination and ionization processes. The study of these energy distributions allows obtaining valuable information about the processes that produce the ions and of the best experimental conditions necessary to produce ion beams with specific kinetic energies for technological applications.
\end{abstract}

Keywords: laser ablation, plasmas, kinetic energies, metal ionizations, ions 


\section{INTRODUCCIÓN}

Las radiaciones de alta intensidad, como las provenientes de láseres pulsados de nanosegundos, interaccionan óptica, mecánica y térmicamente con los materiales y las superficies, provocando su erosión y la emisión de electrones, átomos, moléculas o clusters en estados neutros o ionizados. Todos ellos pueden analizarse para obtener información acerca de los componentes y las características físicas de los materiales (Phipps, 2007; Rubahn, 1999). Además, las especies se generan con intervalos de energía que las hacen muy adecuadas para su utilización en diversas áreas tecnológicas. Así, la ablación de metales con pulsos ultracortos se utiliza para generar nanopartículas (Amoruso et al., 2007).

El interés en todos estos procesos ha favorecido el desarrollo de varias técnicas destinadas a su caracterización. Mientras el análisis de la emisión es la forma más tradicional (Aragón et al., 2005) esta técnica no detecta los valores elevados de la energía cinética con la que son expulsados los iones del plasma. Por ello se han desarrollado técnicas de espectrometría de masas, que permiten observar a los iones, los cuales, junto a los electrones, son las especies más energéticas (Amoruso et al., 1996). Otros trabajos se centran en la observación del cráter formado sobre el metal, permitiendo evaluar la cantidad de material ablacionado y el efecto que la anchura temporal del láser tiene en estos fenómenos (Margarone et al., 2008).

Las técnicas que utilizan la ablación láser se vienen aplicando sobre metales, óxidos y aleaciones para producir haces de partículas (Gammino et al., 2000). Posteriormente, éstos pueden depositarse sobre sustratos apropiados (pulsed laser deposition, PLD) modificando las propiedades del material. Se generan de esta forma películas delgadas de metales, semiconductores y aislantes de alta calidad, películas multicapas, con distintas fases, de superconductores, etc. En general, es posible obtener diferentes tipos de estructuras, composiciones y reactividades, con propiedades que en muchos casos son mejores que las obtenidas por deposición física o química de vapores (PVD/CVD) (Konuma, 1992).

El proceso de ablación en metales comienza con un efecto fotoeléctrico multifotónico que deja una densidad de carga positiva elevada en el metal, provocando una expulsión de iones y otros fragmentos con energías de varios centenares de eV. La interacción de electrones, iones y neutros con la radiación láser desencadena nuevos mecanismos, modificando los grados de ionización y, consecuentemente, acelerando aún más a todas las especies cargadas. El impacto de todas ellas sobre materiales puede provocar su implantación, lo que induce cambios físicos (mecánicos, ópticos, eléctricos, magnéticos, ...) y químicos (reactividad, pasivado, ...) en la superficie. Actualmente las técnicas de implantación se usan de forma rutinaria para modificar superficies y dopar semiconductores (Rose y Ryding, 2006). PLD también puede realizarse en diferentes atmósferas para modificar las especies que desean depositarse. Por ejemplo, la ablación de aluminio en atmósfera de $\mathrm{NH}_{3}$ es eficaz para producir películas de AIN que tienen una gran importancia en el diseño de circuitos electrónicos (Giardini et al., 1997). El conocimiento de la energía cinética y de los mecanismos mediante los cuales estas especies se forman y aceleran, es fundamental para la optimización de estas técnicas.

Los estudios de ablación sobre metales son abundantes pero inconexos, y se encuentran muy dispersos en la literatura. En general, se puede resumir que los datos publicados implican a parámetros que dependen tanto del láser (longitud de onda, fluencia, duración del pulso, ...) como del material (coeficiente de absorción, propiedades térmicas, ...). El análisis de los espectros de emisión (LIBS) permite caracterizar las densidades electrónicas y temperaturas del plasma (Aragón et al., 2005) y es una técnica utilizada en análisis químico (Radziemski, 1994). También es posible analizar los iones producidos por ablación láser recogiendo su espectro de masas en tiempo de vuelo (t.o.f.) (Amoruso et al., 1996, Choi et al., 1999; Torres et al., 2004). Sin embargo, estudios de coincidencias fotón-ión, han demostrado la mayor sensibilidad de las técnicas basadas en la detección de masas (Vadillo et al., 2005).

El objetivo de este trabajo ha sido el análisis de la distribución energética de los iones producidos por ablación láser sobre $\mathrm{Al}, \mathrm{Cu}$ y $\mathrm{Fe}$. Se describe el sistema experimental desarrollado para estas 
determinaciones, se comentan los espectros de masas obtenidos, y se discuten los perfiles de las distribuciones energéticas y el mecanismo que las origina a distintas fluencias láser. La técnica desarrollada permite observar recombinaciones y nuevas ionizaciones por colisión con electrones, explicando las distribuciones de energía cinética con las que se producen los iones en la ablación láser de materiales. Ninguna otra técnica ha mostrado hasta ahora una resolución tan elevada en estas determinaciones.

\section{MATERIALES Y MÉTODOS}

Una descripción detallada de la instrumentación y el método utilizado para estas determinaciones se puede encontrar en trabajos anteriores (Écija et al., 2008); por lo que aquí solo se destacan las características más importantes. Un esquema del sistema experimental se muestra en la figura 1.

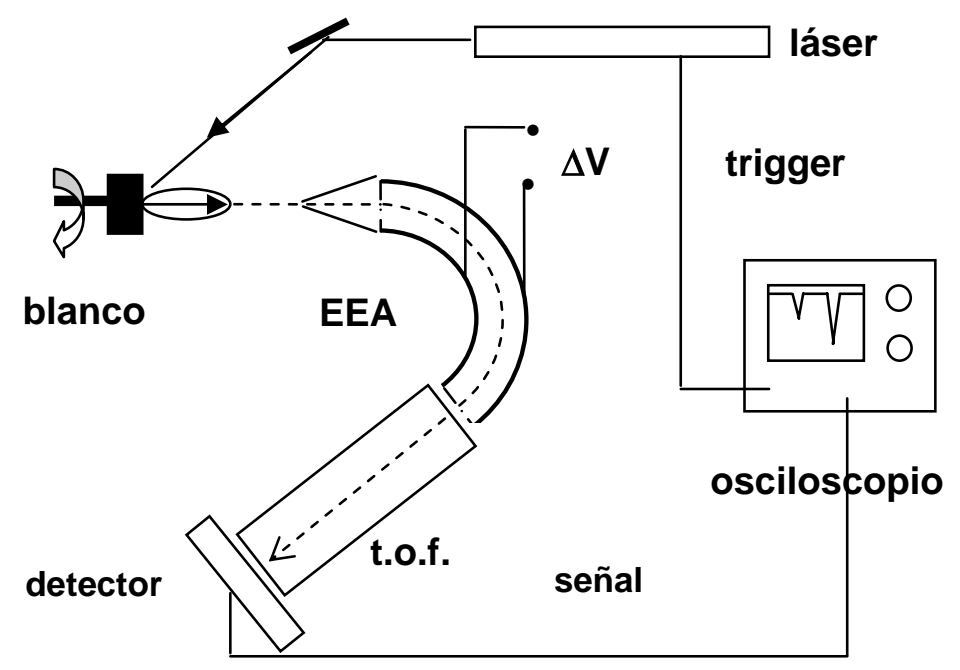

Fig. 1: Esquema del sistema experimental utilizado.

Los metales $\mathrm{Al}$, Cu y Fe, se expusieron a la radiación láser (Nd:YAG, 3 ns/pulso, 450 mJ/pulso a 532 $\mathrm{nm}$, ) en una cámara situada en un vacío de $5 \times 10^{-7} \mathrm{mbar}$. El material se coloca en un soporte giratorio de forma que cada pulso láser encuentra una superficie fresca. La radiación láser focalizada sobre el metal con una lente biconvexa de $50 \mathrm{~cm}$, produjo un punto de luz de unos $0.7 \mathrm{~mm}$ de diámetro. Acercando y alejando dicha lente se consigue variar el diámetro del haz de luz que incide sobre el metal, y con ello la energía depositada sobre la superficie.

Los iones originados en el proceso de ablación emergen en dirección perpendicular al metal, alcanzando una segunda cámara que contiene un analizador electrostático de energías (EEA, Comstock AC-901). Este permite el tránsito de iones con una relación energía/carga seleccionada mediante la aplicación de una diferencia de potencial $(\Delta \mathrm{V})$ entre sus placas de $160^{\circ}(\mathrm{E} / \mathrm{Z}=2,54 . \Delta \mathrm{V})$ (Aragón et al., 1994). El potencial puede variarse desde el umbral (unos pocos voltios) hasta $400 \mathrm{~V}$, energía suficiente para detectar los iones de mayor energía. Finalmente los iones que por tener igual relación E/Z atraviesan el analizador, se separan en un tubo de vuelo (t.o.f.) de $73 \mathrm{~cm}$ antes de alcanzar el detector (placas multicanales en configuración tipo chevron). La señal del detector se envía a un osciloscopio (Tektronix TDS360) y posteriormente al ordenador para su almacenamiento y posterior análisis.

El osciloscopio recibe la orden de detección (trigger) tras la señal del Q-switch procedente del láser. En la figura 2 se muestra como ejemplo el resultado de un espectro de masas, obtenido por ablación láser sobre $\mathrm{Cu}$. La relación $\mathrm{E} / \mathrm{Z}$ es de $170 \mathrm{eV}$ y la fluencia láser $8,6 \mathrm{~J} / \mathrm{cm}^{2}$. Se observan los picos 
originados por los iones $\mathrm{Cu}^{+}, \mathrm{Cu}^{2+}, \mathrm{Cu}^{3+}$ y $\mathrm{Cu}^{4+}$, que corresponden a energías cinéticas de $170 \mathrm{eV}$, $340 \mathrm{eV}, 510 \mathrm{eV}$ y $680 \mathrm{eV}$ respectivamente. Un escaneado del voltaje aplicado al EEA $(\Delta \mathrm{V})$ permite un análisis completo de las distribuciones de energía. El espectro está lo suficientemente resuelto como para que se distingan los dos isótopos del Cu de masas 63 y 65 u.m.a., con una relación entre las intensidades de ambos similar a la que se recoge en la bibliografía (69\% y $31 \%$ respectivamente) (Lide, 2006).

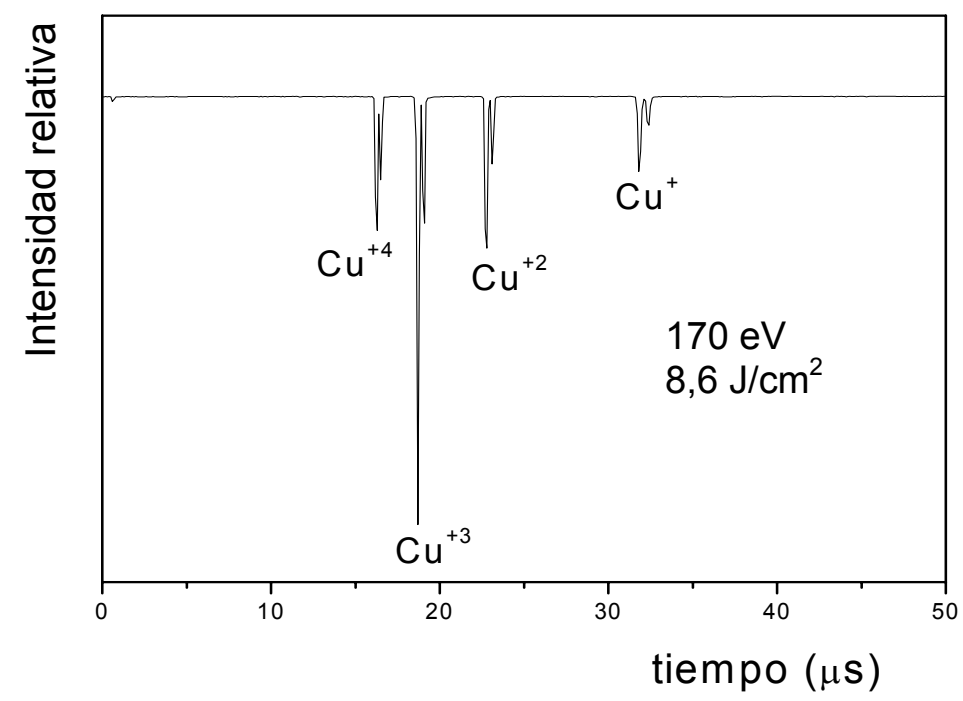

Fig. 2: Ejemplo de espectro de masas en tiempo de vuelo obtenido por ablación láser de Cu.

Los iones se expulsan del metal con energías cinéticas que se ajustan experimentalmente a distribuciones de Maxwell-Boltzmann, con una componente de velocidad debida a la repulsión entre cargas (Torrisi et al., 2002). Estas distribuciones se conocen como de Maxwell-Boltzmann-Coulomb $(\mathrm{MBC})$ :

$F\left(v_{x}\right)=A \cdot\left(\frac{m}{2 \cdot \pi \cdot k \cdot T}\right)^{3 / 2} v_{x}^{3} \cdot \exp \left(-\frac{m \cdot\left(v_{x}-\left(v_{k}+v_{c}\right)\right)^{2}}{2 \cdot k \cdot T}\right)$

Donde $A$ es una constante de normalización, $m$ la masa del ión, $k$.T la temperatura iónica ( $k$ es la constante de Boltzmann), y $v_{k}+v_{c}$ la velocidad que adquieren los iones, suma de la velocidad de expansión $\left(\mathrm{v}_{\mathrm{k}}\right)$ y la de Coulomb $\left(\mathrm{v}_{\mathrm{c}}\right)$ que da cuenta de la aceleración que experimentan en un campo eléctrico intenso. $v_{k}$ se puede determinar como si se tratara de una expansión supersónica (Kelly, 1990):

$v_{k}=\left(\frac{y \cdot k \cdot T}{m}\right)^{1 / 2}$

siendo $\gamma$ la relación de capacidades caloríficas, cuyo valor es de 5/3 para especies monoatómicas. En la ecuación 1 , los términos a evaluar son, además del factor preexponencial $A$, la velocidad media de los iones $v_{k}+v_{c}$ y la temperatura iónica $k . T$.

\section{RESULTADOS Y DISCUSIÓN}

La incidencia sobre un metal de una radiación de alta fluencia y de energía por fotón inferior a su función de trabajo, produce un efecto fotoeléctrico multifotónico. En el caso de los metales Al, Cu y 
Fe, con funciones de trabajo de 4,28 eV, 4,65 eV y 4,5 eV respectivamente (Lide, 2006), es necesaria una absorción mínima de dos fotones de $532 \mathrm{~nm}$ para provocar la emisión de fotoelectrones. Una vez producidos éstos, pueden colisionar con otros átomos y/ó iones y producir nuevas ionizaciones. Todo este proceso ocurre en tiempos muy breves comparados con la anchura temporal del pulso láser ( $3 \mathrm{~ns}$ ) pudiendo existir varias etapas de producción de fotoelectrones, lo que genera una superficie con una región de carga positiva elevada. Esta inestabilidad provoca una explosión coulómbica en la que iones, neutros y electrones son expulsados del material. Se trata de un proceso global muy complejo, ya que las reacciones a las que se ven sometidas todas estas especies son variadas y múltiples: ionizaciones, recombinaciones, desproporcionaciones, etc. La importancia de cada una de ellas depende de las características del metal y de la radiación.

La figura 3 muestra algunos ejemplos de distribuciones de energía cinética junto a sus curvas de ajuste a la ecuación (1) en cada caso. En las figuras $3 a$ y 3 b se observa como se complican las distribuciones al aumentar la fluencia láser en el caso de Al. En las figuras 3c y $3 d$ se muestran dos ejemplos de distribuciones para $\mathrm{Cu}$ y $\mathrm{Fe}$ a fluencias elevadas. En estos casos, junto a la complejidad de la totalidad de las distribuciones, se observa que para cada ión la distribución es posible descomponerla en suma de dos distribuciones MBC, una de ionización y otra de producción directa del ión (ver más abajo). En la tabla 1 se resumen los parámetros de ajuste de estas distribuciones.
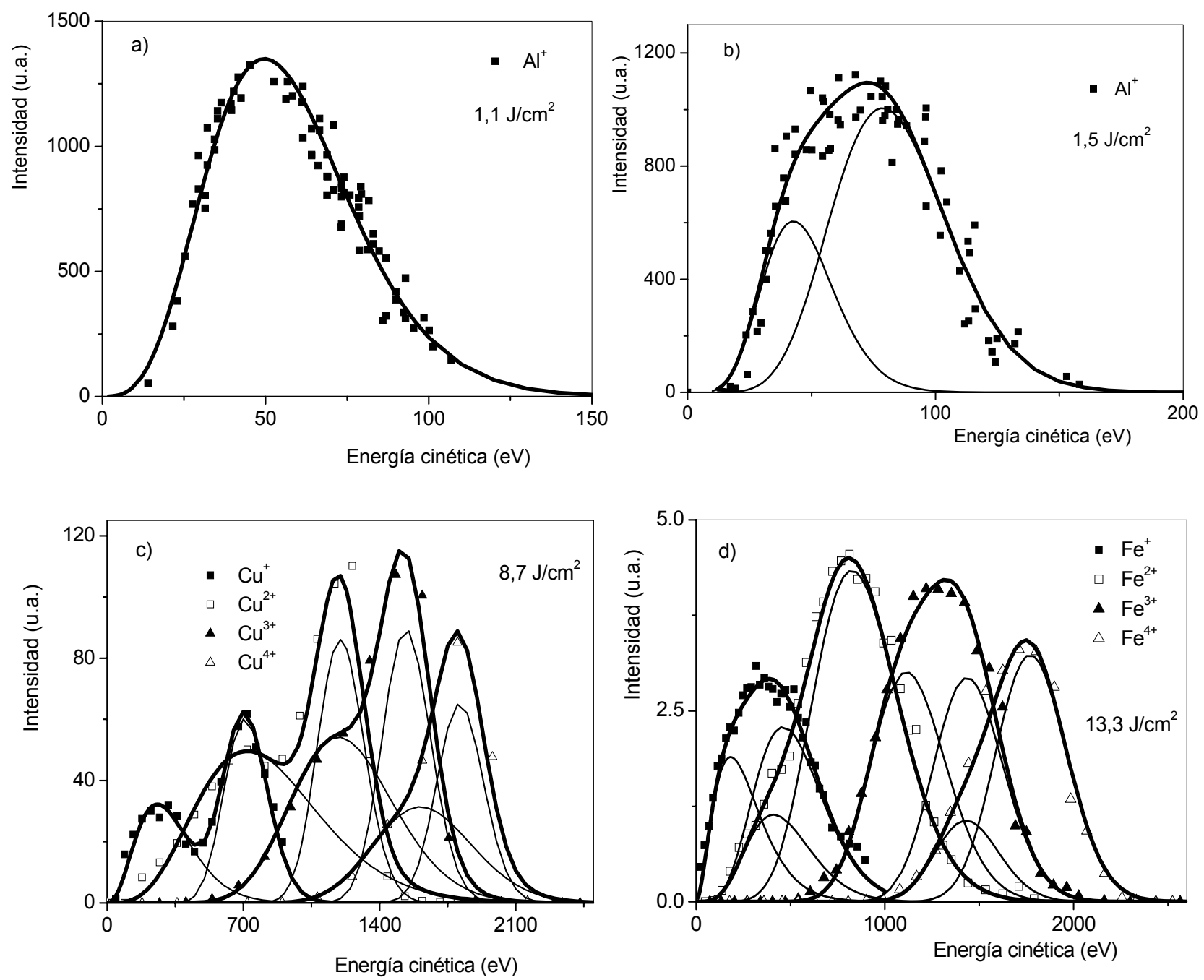

Fig. 3: Distribuciones de energía cinética de los iones observados por ablación láser sobre Al a fluencias de $1,1 \mathrm{~J} / \mathrm{cm}^{2}$ a) y $1,5 \mathrm{~J} / \mathrm{cm}^{2}$ b); Cu a $8,7 \mathrm{~J} / \mathrm{cm}^{2}$ c) y Fe a $13,3 \mathrm{~J} / \mathrm{cm}^{2} \mathrm{~d}$ ). 
Tabla 1: Resumen de resultados de los parámetros de ajuste (ecuación 1) obtenidos para las distribuciones de energía cinética que se muestran en la figura 3.

\begin{tabular}{|c|c|c|c|c|c|}
\hline \multicolumn{2}{|c|}{ Fluencia $\left(\mathrm{J} / \mathrm{cm}^{2}\right)$} & \multirow{2}{*}{$\frac{\text { lón observado }}{\mathrm{Al}^{+}}$} & \multirow{2}{*}{$\frac{\text { Origen del ión }}{\mathrm{Al}^{+}}$} & \multirow{2}{*}{$\frac{\mathbf{v}_{\mathbf{k}}+\mathbf{v}_{\mathbf{c}}(\mathbf{m} / \mathbf{s})}{1,58.10^{4}}$} & \multirow{2}{*}{$\frac{\text { k.T }(e V)}{5,5}$} \\
\hline a) & 1,1 & & & & \\
\hline \multirow[t]{2}{*}{ b) } & \multirow[t]{2}{*}{1,5} & \multirow[t]{2}{*}{$\mathrm{Al}^{+}$} & $\mathrm{Al}^{0}$ & $1,79.10^{4}$ & 5 \\
\hline & & & $\mathrm{Al}^{+}$ & $2,39.10^{4}$ & 2 \\
\hline \multirow[t]{8}{*}{ c) } & \multirow[t]{8}{*}{8,7} & \multirow[t]{2}{*}{$\mathrm{Cu}^{+}$} & $\mathrm{Cu}^{0}$ & $3,05.10^{4}$ & 50 \\
\hline & & & $\mathrm{Cu}^{+}$ & $6,97.10^{4}$ & 9 \\
\hline & & \multirow[t]{2}{*}{$\mathrm{Cu}^{2+}$} & $\mathrm{Cu}^{+}$ & $6,27.10^{4}$ & 70 \\
\hline & & & $\mathrm{Cu}^{2+}$ & $9,07.10^{4}$ & 6 \\
\hline & & \multirow[t]{2}{*}{$\mathrm{Cu}^{3+}$} & $\mathrm{Cu}^{2+}$ & $8,87.10^{4}$ & 30 \\
\hline & & & $\mathrm{Cu}^{3+}$ & $10,4 \cdot 10^{4}$ & 5 \\
\hline & & \multirow[t]{2}{*}{$\mathrm{Cu}^{4+}$} & $\mathrm{Cu}^{3+}$ & $10,5.10^{4}$ & 20 \\
\hline & & & $\mathrm{Cu}^{4+}$ & $11,3.10^{4}$ & 4 \\
\hline \multirow[t]{8}{*}{ d) } & \multirow[t]{8}{*}{13,3} & \multirow[t]{2}{*}{$\mathrm{Fe}^{+}$} & $\mathrm{Fe}^{0}$ & $1,18.10^{4}$ & 65 \\
\hline & & & $\mathrm{Fe}^{+}$ & $3,48.10^{4}$ & 40 \\
\hline & & \multirow[t]{2}{*}{$\mathrm{Fe}^{2+}$} & $\mathrm{Fe}^{+}$ & $3,38.10^{4}$ & 33 \\
\hline & & & $\mathrm{Fe}^{2+}$ & $5,02.10^{4}$ & 40 \\
\hline & & \multirow[t]{2}{*}{$\mathrm{Fe}^{3+}$} & $\mathrm{Fe}^{2+}$ & $6,05.10^{4}$ & 18 \\
\hline & & & $\mathrm{Fe}^{3+}$ & $6,96.10^{4}$ & 12 \\
\hline & & \multirow[t]{2}{*}{$\mathrm{Fe}^{4+}$} & $\mathrm{Fe}^{3+}$ & $6,96.10^{4}$ & 10 \\
\hline & & & $\mathrm{Fe}^{4+}$ & $7,76.10^{4}$ & 10 \\
\hline
\end{tabular}

Las incertidumbres asociadas a estas determinaciones dependen de diversas variables, unas del láser y otras del EEA que es menos preciso a voltajes más bajos. Los estudios realizados sobre estos metales y otros, permiten estimar un error de 5-7\% en la determinación de energías cinéticas. Todas las distribuciones observadas presentan características similares. A bajas fluencias del láser, se ajustan a una distribución MBC simple; pero, a medida que aumenta la fluencia, las distribuciones se complican, debiendo describirse como suma de dos distribuciones de MBC. En estos casos el máximo secundario de la distribución se sitúa a una energía cinética similar a la del máximo principal del ión de carga inmediatamente inferior (Z-1). La mayor componente de la velocidad de los iones es la velocidad de Coulomb, que tiene su origen en las interacciones electrostáticas de los iones en la superficie del metal. Los iones de mayor carga son en consecuencia más acelerados. El hecho de que un aumento de fluencia provoque la aparición de máximos secundarios a energías más bajas, debe de relacionarse con procesos en los que el ión de carga (Z-1) sufre un proceso de ionización tras colisionar con un electrón. En esta colisión, debido a la enorme diferencia de masas entre el electrón y el ión, no se produce apenas transferencia de energía cinética, sino una ionización en la que el nuevo ión de carga $Z$ mantiene la energía del ión precursor de carga (Z-1) y, por lo tanto, aparece en el espectro de masas junto a los iones de carga $Z$ pero a menores energías. Por esta razón, en la tabla 1 se recogen dos orígenes distintos para cada distribución de energía cinética, que así se expresa como suma de dos distribuciones de MBC. 
Una conclusión importante de esta discusión, es que se puede relacionar las distribuciones de energía más baja que aparecen junto a los iones de carga +1 , como pertenecientes a los neutros, y así estimar su densidad. En los ejemplos que se muestran en la figura 3, y cuyos parámetros de ajuste se recogen en la tabla 1, las distribuciones de energía de los fragmentos neutros de $\mathrm{Al}^{0}, \mathrm{Cu}^{0} \mathrm{y}$ $\mathrm{Fe}^{0}$ pueden observarse en las figuras b), c) y d) junto a las del $\mathrm{Al}^{+}, \mathrm{Cu}^{+}$y $\mathrm{Fe}^{+}$respectivamente. Las velocidades de estos neutros son $1,79.10^{4} ; 3,05.10^{4}$ y $1,18.10^{4} \mathrm{~m} / \mathrm{s}$. El resto de velocidades va aumentando paulatinamente con el grado de ionización. Así, en las figuras $3 c$ y $3 \mathrm{~d}$, donde el grado de ionización observado es hasta de +4 para el $\mathrm{Cu}$ y el $\mathrm{Fe}$, se observa que la velocidad de los iones aumenta con su grado de ionización, prueba del proceso de aceleración que sufren en el seno del plasma por el campo eléctrico creado. Estos iones son los que van a tener más facilidad para implantarse en un soporte o incluso para inducir reacciones químicas (Rose y Ryding, 2006).

Además de los procesos de ionización, un estudio de la variación de la población de los iones con la energía cinética permite observar las recombinaciones que se producen entre ellos. En la figura 4 se muestra como ejemplo la variación relativa de población del ión $\mathrm{Al}^{\mathrm{Z+}}$ en un plasma de $\mathrm{Al}$ generado a una fluencia láser de $5,0 \mathrm{~J} / \mathrm{cm}^{2}$.

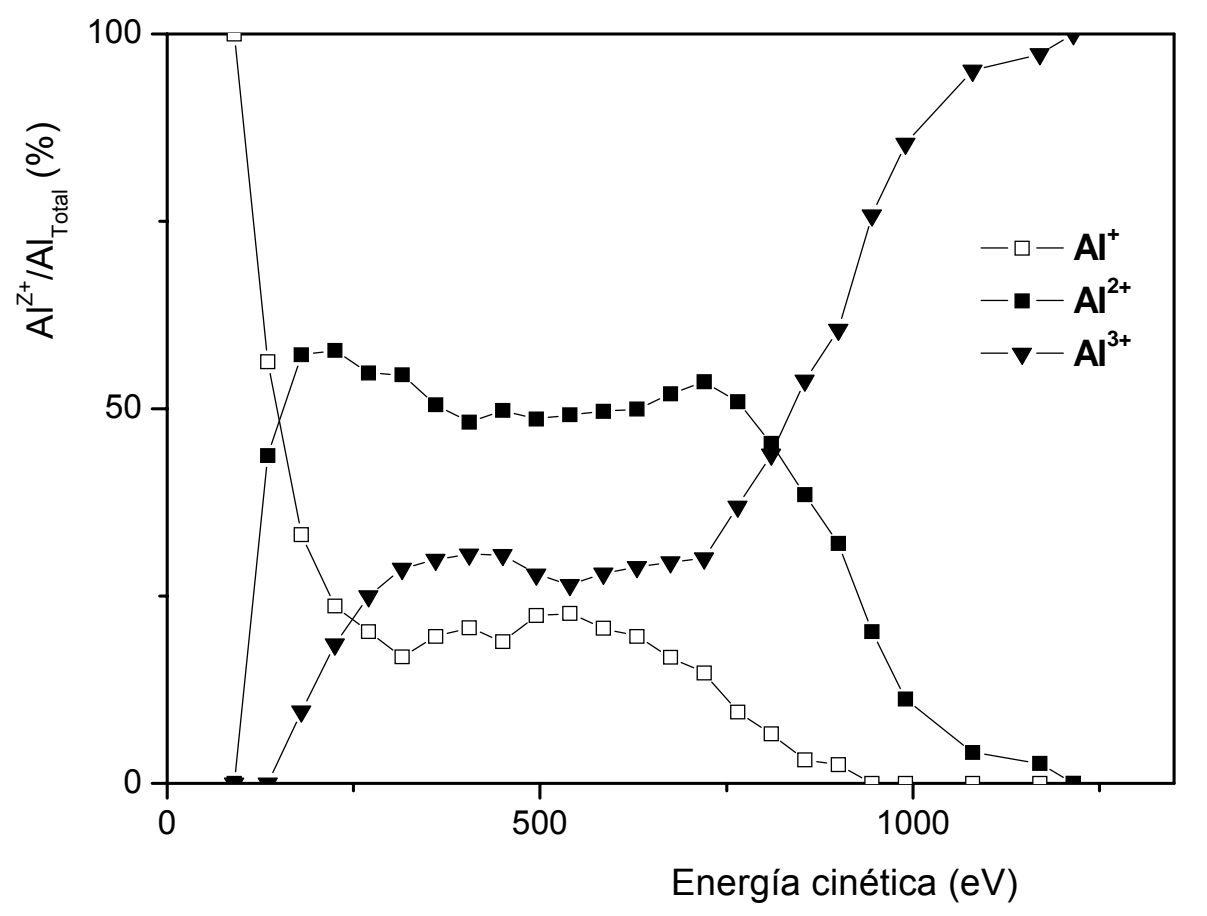

Fig. 4: Población relativa de iones $\mathrm{Al}^{\mathrm{Z+}}$ en una pluma de Aluminio producida por ablación láser a una fluencia de $5,0 \mathrm{~J} / \mathrm{cm}^{2}$.

El umbral de aparición para el $\mathrm{Al}^{2+}$ indica que cuando se forma es, en parte, debido a la desaparición de $\mathrm{Al}^{+}$. Esto parece corroborarse por el hecho de que al desaparecer el ión $\mathrm{Al}^{+}$también comienza el descenso de la población del $\mathrm{Al}^{2+}$. Además, en el intervalo de energías donde $\mathrm{Al}^{+}, \mathrm{Al}^{2+}$ y $\mathrm{Al}^{3+}$ coexisten, la producción de $\mathrm{Al}^{2+}$ es superior a la de $\mathrm{Al}^{+}$y $\mathrm{Al}^{3+}$ alcanzando el valor máximo en el umbral de energías donde surge el $\mathrm{Al}^{3+}$. Durante todo ese intervalo de energías los tres iones tienen un comportamiento plano similar, produciéndose un rápido incremento de la población de iones $\mathrm{Al}^{3+}$ cuando los $\mathrm{Al}^{+}$comienzan a desaparecer. Estas observaciones parecen indicar que hay una interconexión entre los iones analizados y que los procesos $\mathrm{Al}^{+}+\mathrm{hv} \rightarrow \mathrm{Al}^{2+}+\mathrm{e}^{-}$y $\mathrm{Al}^{2+}+\mathrm{hv} \rightarrow \mathrm{Al}^{3+}+\mathrm{e}^{-}$son importantes en este intervalo de energías.

Conclusiones similares de correlación entre diferentes especies iónicas, se pueden obtener de un análisis de poblaciones relativas para otros casos de ablación, en otros metales y a otras fluencias. 


\section{CONCLUSIONES}

Este trabajo muestra una técnica de espectrometría de masas en tiempo de vuelo, que permite medir las velocidades de los iones producidos por ablación láser sobre metales. La técnica es lo suficientemente sensible para ser utilizada como herramienta de análisis de superficies y materiales, compitiendo con LIBS. Todas las distribuciones se han ajustado adecuadamente a distribuciones de Maxwell-Boltzmann con una componente coulómbica. Así, se han determinado las velocidades de expansión y de Coulomb además de la temperatura iónica en cada caso. Las velocidades de los iones estudiados están en la región de $10^{4} \mathrm{~m} / \mathrm{s}$, siendo los de mayor carga los más acelerados por el intenso campo eléctrico que se genera en el plasma. Dentro de un mismo metal, un aumento de la fluencia del láser provoca un incremento en la velocidad de todos los iones, pudiéndose utilizar esta variable para alcanzar velocidades iónicas adecuadas para realizar PLD o implantación de iones.

Un estudio detallado de las distribuciones de velocidad a fluencias altas, indica que éstas no corresponden a distribuciones simples de MBC, apareciendo bandas laterales que se relacionan con procesos donde tienen importancia los electrones del plasma: ionizaciones y recombinaciones. En el caso de las distribuciones de energía de los iones $\mathrm{Al}^{+}, \mathrm{Cu}^{+}$y $\mathrm{Fe}^{+}$, sus bandas laterales se corresponden con las que tienen los átomos neutros $\mathrm{Al}^{0}, \mathrm{Cu}^{0}$ y $\mathrm{Fe}^{0}$, que han sufrido una ionización por los electrones del plasma. Estas especies son detectadas a tiempos de vuelo característicos de los iones de carga +1 , pero mantienen la información cinética de sus neutros de origen. Finalmente, también se ha observado la importancia que tienen en el plasma de ablación las reacciones que conectan a los distintos iones: recombinaciones entre iones, nuevas ionizaciones, etc. Todos estos procesos muestran la complejidad, interconexión y elevado número de procesos que tienen lugar en los plasmas de ablación.

\section{AGRADECIMIENTOS}

Al Ministerio de Educación y Ciencia (Madrid, España) por la concesión de un proyecto Consolider. Al Gobierno Vasco (Vitoria-Gasteiz, España) y a la Universidad del País Vasco (UPV/EHU) por la financiación de este trabajo.

\section{REFERENCIAS}

Amoruso, S., y otros cinco autores; High fluence laser ablation of aluminium targets: Time-of-flight mass analysis of plasmas produced at wavelenghts 532 and $355 \mathrm{~nm}$, Appl. Phys. A: 62(6), 533-541 (1996).

Amoruso, S. y otros cuatro autores; An analysis of the dependence on photon energy of the process of nanoparticles generation by femtosecond laser ablation in a vacuum, Nanotechnology: 18(14), 145612/1-145612/6 (2007).

Aragón, C.; J.A. Aguilera, F. Blanco y J. Campos; A simple and compact system for energy, charge and mass analysis of ions formed in laser-produced plasmas, Vacuum: 45(9), 923-927 (1994).

Aragón, C.; F. Peñalba y J.A. Aguilera; Curves of growth of neutral atom and ion lines emitted by a laser induced plasma, Spectrochim. Acta, Part B: Atom.: 60, 879-887 (2005).

Choi, Y.-K., H.-S. Im y K.-W. Jung; Temporal evolution of laser-ablated $\mathrm{Co}^{+}$ions probed by time-offlght mass spectrometry, Appl. Surf. Sci.: 150 (1-4), 152-160 (1999).

Ecija, P., y otros seis autores; Fundamental processes in nanosecond pulsed-laser ablation of metals in vacuum, Phys. Rev. A: 77(3) 032904/1-032904/8 (2008).

Gammino, S., y otros nueve autores; Preliminary tests for the electron cyclotron resonance ion source coupled to a laser ion source for charge state enhancement experiment, Rev. Sci. Instrum.: 71(2, Pt.II), 1119-1121 (2000). 
Giardini, G., y otros 5 autores; AIN thin film deposition by pulsed laser ablation of $\mathrm{Al}$ in $\mathrm{NH}_{3}$, Thin Solid Films: 295(1-2), 77-82 (1997).

Kelly, R.; On the dual role of the Knudsen layer and unstable, adiabatic expansion in pulse sputtering phenomena; J. Chem. Phys.: 92(8), 5047-5056 (1990).

Konuma, M.; Film Deposition by plasma techniques, pp 107-125, Springer-Verlag, Berlin, Germany (1992).

Lide, D.R.; Handbook of Chemistry and Physics, 87 ed., CRC Press, Boca Raton, FL (2006).

Margarone, D. y otros catorce autores; Studies of craters' dimension for long-pulse laser ablation of metal targets at various experimental conditions, Appl. Surf. Sci.: 254(9), 2797-2803 (2008).

Phipps, C.R.; Laser ablation and its applications, pp 281-373, Springer Science, New York, USA (2007).

Radziemski, L.J.; Review of selected analytical applications of laser plasmas and laser ablation, 1987-1994, Microch. J.: 50, 218-234 (1994).

Rose, P.H. y G. Ryding; Concepts and designs of ion implantation equipment for semiconductor processing, Rev. Sci. Instr.: 77(11), 111101/1-111101/12, (2006).

Rubahn, H.-G.; Laser applications in surface science and technology, pp215-276, John Wiley\&Sons, West Sussex, England (1999).

Torres, R., M. Jadraque y M. Martin; Cluster formation and laser-induced effects in the ablation of 2 $\mathrm{Cu}\left(\mathrm{CO}_{3}\right) . \mathrm{Cu}(\mathrm{OH})_{2}$. Time-of-flight mass spectrometric study, Appl. Phys. A: 79(4-6), 1057-1060 (2004).

Torrisi, L., Gammino, S., Andò y L. Làska; Tantalum ions produced by 1064 nm pulsed laser irradiation, J. Appl. Phys.: 91(7), 4685-4692 (2002).

Vadillo, J.M., C.C. García, J.F. Alcántara y J.J. Laserna; Termal-to-plasma transitions and energy thresholds in laser ablated metals monitored by atomic emission/mass spectrometry coincidence analysis, Spectrochim. Acta, Part B: Atom. Spectrosc.: 60 (7-8), 948-954 (2005). 
